ARTIGO ORIGINAL

\title{
PERFIL EPIDEMIOLÓGICO E ÓBITOS EM PACIENTES INTERNADOS COM DOENÇA REUMÁTICA CRÔNICA DO CORAÇÃO ENTRE 2011 A 2018 NO ESTADO DE TOCANTINS.

\author{
EPIDEMIOLOGICAL PROFILE AND DEATHS IN PATIENTS WITH \\ CHRONIC RHEUMATIC HEART DISEASE AMONG 2011 TO 2018 IN
} THE STATE OF TOCANTINS.
}

\section{G ACESSO LIVRE}

Citação: Figueira $\mathrm{CB}$, Dias BCS, Costa CR, Martins KLM, Silva MB, Ruela MV, D'alessandro WB (2020), PERFIL EPIDEMIOLÓGICO E ÓBITOS EM PACIENTES INTERNADOS COM DOENÇA REUMÁTICA CRÔNICA DO CORAÇÃO ENTRE 2011 A 2018 NO ESTADO DE TOCANTINS 10(4):.

\section{Instituição:}

1 Acadêmica do Curso de Medicina Universidade Gurupi, Gurupi, Tocantins, Brasil.

2 Professor Doutor em Biomedicina Universidade de Gurupi, Gurupi, Tocantins, Brasil.

Autor correspondente: Caroline Barros Figueira;

carolinebarrosfigueira@gmail.com

Editor: Carvalho A. A. B. Medicina, Universidade Federal do Tocantins, Brasil.

Publicado: 18 de outubro de 2020.

Direitos Autorais: (c) 2020 Peixoto et al. Este é um artigo de acesso aberto que permite o uso, a distribuição e a reprodução sem restrições em qualquer meio, desde que o autor original e a fonte sejam creditados.

Conflito de interesses: os autores declararam que não existem conflitos de interesses.
Caroline Barros Figueira ${ }^{1}$, Brenda Caroline da Silveira Dias ${ }^{1}$,Carolina Rodrigues Costa ${ }^{1}$, KarolLayne Macena Martins ${ }^{1}$, Manuela Bandeira da Silva ${ }^{1}$, Marina Vieira Ruela ${ }^{1}$, Walmirton Bezerra D'alessandro 2

\begin{abstract}
RESUMO
Introdução: A cardiopatia reumática crônica é uma complicação não supurativa por infecção da faringoamigdalite pelo streptococcus b-hemolítico do grupo A. Ocorre em indivíduos genéticamente susceptíveis e que tiveram uma resposta imune tardia. É a manifestação crônica mais severa da febre reumática, que se caracteriza por fibrose e calcificação valvar. É uma patologia de alta incidência no Tocantins, mas que tem poucos estudos que informam sobre o perfil do paciente e sua relevância, algo fundamental para a prevenção dos agravos. O objetivo desse trabalho foi descrever as características sociodemográficas e epidemiológicas dos pacientes internados em Hospitais do SUS entre 2011 a 2018 no Estado de Tocantins. Método: Estudo epidemiológico, descritivo, retrospectivo com uma população (n) de 268 pacientes admitidos entre 2011 e 2018 no eEstado do Tocantins. Os dados foram coletados da base de dados do Departamento de Informática do Sistema Único de Saúde (DATASUS). Foram analisadas as características epidemiológicas como sexo, raça/cor, faixa etária, média de permanência e taxa de mortalidade. Resultados: No período analisado de 2011 a 2018, as doenças do aparelho circulatório com ênfase na doença reumática crônica do coração, teve maior prevalência o ano de 2012, n=56, o que equivale 20,8\% de um total de 268 internações. Dentre todas as faixas etárias analisadas, menor que 1 ano até maior que 80 anos, a idade de maior predomínio foi de $40-49$ anos, $n=49$, correspondente a $18,2 \%$ do total analisado. De todas as raças apuradas, a parda foi dominante, $n=189$, com $70,5 \%$. O maior número de óbitos foi em 2014, tendo $33,3 \%, n=4$ de um total de 12 óbitos do período estudado. Conclusões: A incidência de internação hospitalar foi elevada quando comparada à média da região Norte. A descrição das características sociodemográficas e epidemiológicas norteia o planejamento das ações dos profissionais de saúde para fornecer um atendimento de melhor qualidade. $\mathrm{O}$ estudo apresentou limitações visto que a fontes de dados (SIH/SUS) registra somente informações realizadas no sistema público de saúde. Ressalta-se que o tratamento da febre reumática para ser eficiente deve ser precoce e se possível, antes do comprometimento do coração.
\end{abstract}

Palavras-chave: Epidemiologia, Doença reumática, Saúde pública, Cardiopatia reumática.

\begin{abstract}
Introduction: Chronic rheumatic heart disease is a complication of non-suppurative infection of the pharyngotonsillitis by b-hemolytic Streptococcus Group A, occurs in genetically susceptible individuals and had a late immune response. Is the most severe chronic manifestation of rheumatic fever, which is characterized by fibrosis and valvular calcification. Is a pathology of high incidence in Tocantins, but that has very few studies that inform about the patient profile and your relevance, something essential for the prevention of aggravations. The objective of this work was to describe the socio-demographic and epidemiological characteristics of patients admitted to Hospitals of the SUS between 2011 to 2018 in the State of Tocantins. Material and Methods: Epidemiological study, descriptive and retrospective with a population of 265 patients admitted between 2011 to 2018 in the Tocantins State. The data were collected from the Department of Informatics of the unified health system (DATASUS). Epidemiological characteristics were analysed by sex, race/color, age range, average of permanence and mortality rate. Results: fifty nine comma sixty two percent were female, $71,32 \%$ race/brown color and the most prevalent age group was 40 to 49 years $(17,73 \%)$ and the average stay of hospitalization of patients with chronic rheumatic disease was 10.5 days. The in Hospital death rate was $4.52 \%$. Conclusion: The incidence of hospitalization was elevated when compared to the average in the northern region. The description of the socio-demographic and epidemiological features guides the planning of health professionals to provide a service a better quality service. The study presented limitations since the datasources (SIH/SUS) records only information held in the public health system. It should be noted that the treatment of rheumatic fever to be effective must be early and if possible, before the impairment of the heart.

Keywords: Epidemiology; Infections; Rheumatic disease, Public health, Rheumatic Heart.
\end{abstract}


INTRODUÇÃO

A cardiopatia reumática crônica é causada pelo streptococcus b-hemolítico do grupo A de Lancefield, sendo uma complicação não supurativa por infecção da faringoamigdalite. Ocorre em indivíduos geneticamente predispostos e que tiveram uma resposta imune demorada. Há maior incidência entre 5 e 15 anos de idade. ${ }^{1}$

Após 3 semanas da infecção, algumas sintomatologias da febre reumática aguda podem se manifestar no cérebro, articulações, coração e a pele. 0 envolvimento cardíaco decorre do processo inflamatório que atinge o endocárdio, o miocárdio e o pericárdio. É a manifestação crônica mais severa advinda da febre reumática, que se caracteriza por fibrose e calcificação valvar nessas camadas, acarretar deformidades estruturais nas valvas cardíacas. Sendo mais frequente a danificação isolada da valva mitral seguida da valva aórtica. ${ }^{1}$

A cardiopatia reumática integra uma das elementares adversidades de saúde pública do Brasil. Além disso, trata-se de uma enfermidade que acarreta elevado gasto para os serviços de saúde diligenciando frequentes hospitalizações, com predomínio de indicação da cirurgia cardíaca. $^{2}$

Os sintomas da cardiopatia reumática subordinam-se ao molde de lesão valvar e da valva agredida, pode externar anos ou décadas depois do primeiro incidente de febre reumática. Os portadores de valvopatia mitral tem como objeções frequentes as palpitações, enquanto em pacientes com valvopatia aórtica costumam relatar dor torácica e síncope aos esforços. ${ }^{2}$

O quadro clínico mais recorrente das disfunções valvares é a insuficiência cardíaca que se mostra com fadiga, hemoptise, cansaço ao se deitar, cansaço aos esforços, chiado, edema periférico e tosse. A cardiopatia reumática também constitui um fator predisponente para endocardite infecciosa, principalmente em pacientes com próteses valvares. ${ }^{2}$

A vasculite verrucosa com edema e deformidade do tecido valvular com espessamento fibroso e fusão das comissuras valvares, levando a graus variados de insuficiência ou estenose configura a endocardite reumática, já a inflamação das camadas do pericárdio e derrame identifica a pericardite reumática. ${ }^{3}$

A taxa de óbito intra-hospitalar por doença reumática crônica do coração em pacientes internados pelo SUS foi de 4,52\% referente aos anos de 2011 a 2018, totalizando um total de 12 óbitos em relação aos 268 portadores da doença reumática crônica. Com um gasto no tratamento clínico cerca de $2.228 .300,04$ milhões de reais nesse período.

Os critérios de Jones, serve como um guia para o diagnóstico do quadro clínico, tendo as manifestações cardiológicas (cardite, pericardite) como critérios maiores que sugerem confirmar a patologia. Ressalta-se que a Doença reumática tem manifestações atípicas que podem não satisfazer esses critérios. ${ }^{3}$

A doença reumática crônica do coração tem alta incidência no Tocantins, mas tem poucos estudos que informam sobre o perfil do paciente e sua relevância, algo fundamental para a prevenção dos agravos. Deste modo, o objetivo deste trabalho foi descrever as características sociodemográficas e epidemiológicas dos pacientes internados em Hospitais do SUS entre 2012 a 2018 no Estado de Tocantins e discutir as informações no bojo dos dados encontrados, para possibilitar formas de prevenção e o direcionamento das políticas públicas para sanar a patologia.

\section{MÉTODO}

Trata-se de um estudo epidemiológico, descritivo e transversal realizado por meio da coleta de dados de domínio público disponibilizados pelo Ministério da Saúde do Brasil, através da plataforma online do Departamento de Informática do Sistema Único de Saúde (DATASUS). Para o levantamento dos dados, foram acessados os Sistemas de Informações Hospitalares e sobre Mortalidade (SIM/SIH) em um recorte temporal de 7 anos, compreendido o período de 2012 a 2018.

As variáveis epidemiológicas utilizadas foram: total de internações no período citado utilizado o filtro por região, destacando a região norte, acrescida das variáveis de raça/cor, faixa etária e óbitos utilizando o filtro por estado, destacando o estado do Tocantins. Os números foram tabulados em de planilhas eletrônicas do programa Microsoft Excel e analisados através de estatísticas descritivas.

Por tratar-se de dados públicos, disponíveis online, sem identificação pessoal ou institucional, não foi necessária aprovação do estudo em Comitê de Ética. A pesquisa não tem conflito de interesses.

\section{RESULTADOS}

No período analisado de 2012 a 2018, as doenças do aparelho circulatório com ênfase na doença reumática crônica do coração, teve maior prevalência o ano de $2012, n=56$, o que equivale $20,8 \%$ de um total de 268 internações.

Gráfico 1. Internações e óbtidos em pacientes com Doença Reumática Crônica do Coração.

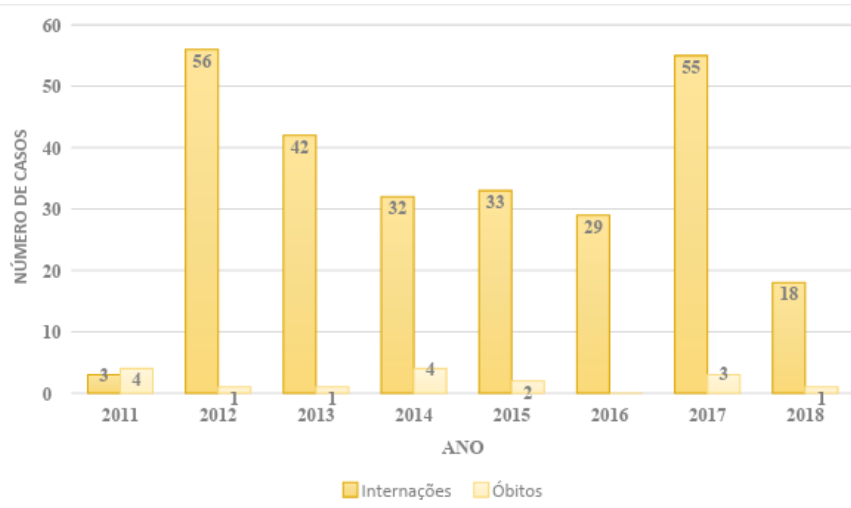

* Não houve dado, pelo sistema DATASUS, referente ao ano de 2016 para notificar a quantidades de óbitos.

Dentre todas as faixas etárias analisadas, menor que 1 ano até maior que 80 anos, a idade de maior predomínio foi de 40-49 anos, $n=49$, correspondente a $18,2 \%$ do total analisado. De todas as raças apuradas, a parda foi dominante, $n=189$, com $70,5 \%$. O número de óbitos foi prevalente nos 
anos 2014 e 2011, tendo ambos 25\%, n=4 de um total de 16 Apesar do recrudescimento da (FR) nos países desenvolvidos, óbitos do período estudado (Gráfico 1 )

Gráfico 2. Comparação entre gêneros em pacientes com doença reumática crônica do coração.

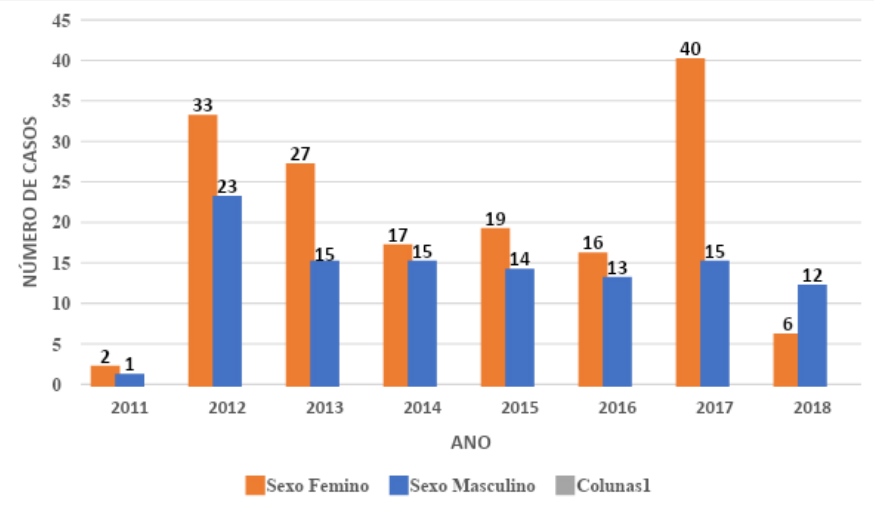

Em relação ao sexo, obteve maior incidência no sexo feminino em 2016, $n=-13$, apresentando $12,04 \%$, de 108 . No que tange ao sexo feminino observou-se que no ano de 2017 apresentou-se prevalente com $25 \%$ de um total de 160 correspondente a todo o período analisado, $(n=40)$. Apenas em 2018 o sexo masculino $(4,4 \%)$ teve maior prevalência sobre o feminino (2,2\%), de um total de 268 casos (Gráfico 2).

Gráfico 3. Incidência de raças acometidas em pacientes com doença reumática crônica do coração.

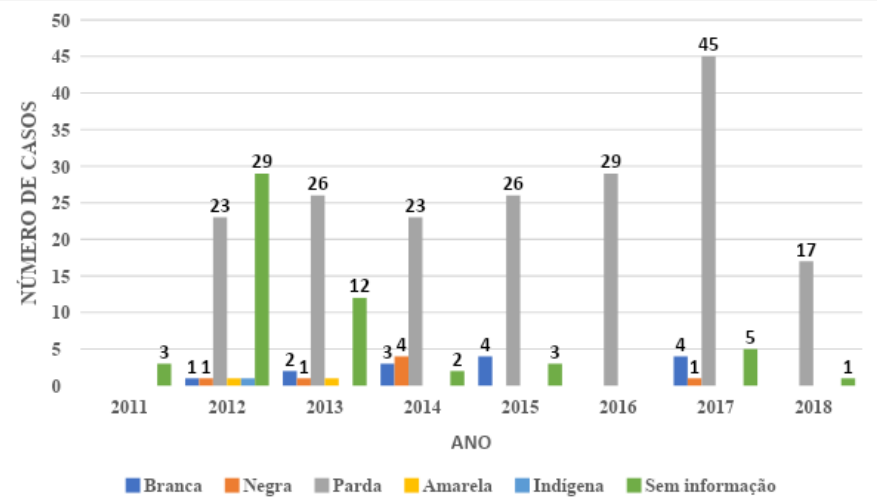

A raça parda tem maior incidência na comparação geral, correspondendo a 70,5 \% dos casos totais. Sendo dominante dos anos de 2013 a 2018, e fica em segundo lugar no ano de 2012 devido ao grande número de casos que não informaram a raça (Gráfico 3).

\section{DISCUSSÃO}

A febre reumática (FR) acomete vários tecidos e órgãos, como coração, vasos, articulações, pele e sistema nervoso, mas sua importância maior reside no fato de poder provocar lesões cardíacas residuais, principalmente, nas valvas mitral e aórtica, sendo a insuficiência mitral o sinal mais comum e, mesmo, cardiopatia aguda grave com curso fatal. É compreensível que a melhor maneira de evitar lesão cardíaca reumática é impedindo a instalação da doença através do tratamento adequado das IVAS (Infecções das Vias Aéreas Superiores) de origem estreptocócica ${ }^{5}$. em particular nos Estados Unidos, a partir da segunda metade da década de 1980, é nos países em desenvolvimento como o Brasil que esta doença está associada à alta morbimortalidade. Um baixo nível socioeconômico-cultural, condições de vida precárias, serviços de atenção primária à saúde inadequados e o tratamento tardio e inadequado das infecções das vias aéreas possivelmente representam os fatores que levam a esse quadro desfavorável, logo é esse atraso no diagnóstico que leva tal doença a ser diagnóstica na fase adulta, como ocorreu no presente trabalho, levando assim a uma cardiopatia reumática crônica (CRC), manifestadas 20 a 40 décadas depois, por isso a faixa etária mais acometida esteve entre 40-49 anos. ${ }^{4}$

Em relação aos principais critérios de Jones, que são os critérios usados para o diagnóstico correto dessa patologia, a artrite esta presente nos pacientes como principal manifestação, a cardite em segundo lugar e a coréia em terceiro. Posteriores vem o eritema marginado e os nódulos subcutâneos todos esses como critérios maiores. Com relação aos critérios menores de Jones, a febre é preponderante pacientes, depois vem a artralgia e o aumento da taxa de sedimentação sanguínea (VHS). Também níveis elevados de anticorpos antistreptocócicos detectados com antiestreptolisina-O são observados ${ }^{4}$. Dois sinais maiores ou 1 maior e 2 menores são necessários para definir o diagnóstico. Entretanto, estes critérios servem como guia, não substituindo o bom senso do médico, desde que várias situações clínicas podem preencher os critérios de Jones e fornecer um diagnóstico falso de $\mathrm{FR}^{5}$.

$E$ alguns pacientes apresentaram pelo menos uma recorrência da FR se não tratada adequadamente ${ }^{4}$ A Organização Mundial da Saúde (OMS) recomenda que tanto o surto inicial quanto as recorrências de $F R$ possam ser prevenidas por meio de estratégias terapêuticas voltadas para o tratamento adequado de infecções estreptocócicas, ou seja, por meio da profilaxia primária, e é, feita preferencialmente com a penicilina $G$ benzatina.. É importante ressaltar que a OMS recomenda que essas estratégias sejam fáceis de executar e de baixo custo. Assim, é urgentemente necessário identificar estratégias governamentais responsáveis para oferecer à população um tratamento imediato e eficaz de infecções por estreptococos da garganta, em conjunto com programas de apoio à profilaxia secundária de FR. Além disso, deve-se determinar se a profilaxia secundária com defeito resulta da ineficácia das abordagens atuais e/ou da falta de adesão ao tratamento ${ }^{4}$.

Com relação à profilaxia primária, uma grande dificuldade deve-se aos quadros subclínicos ou assintomáticos de IVAS, impossibilitando essa profilaxia. Outro problema também observado diz respeito ao tratamento inadequado das IVAS com antibióticos bacteriostáticos, ou mesmo uso de antibióticos bactericidas por período inferior a 10 dias. Quanto à profilaxia secundária, a aderência à medicação por período prolongado é o grande desafio, pois, a penicilina $\mathrm{G}$ benzatina, droga profilática de escolha, tem o inconveniente da dor decorrente de sua aplicação

Como citado essa é uma doença que acomete mais crianças e jovens adultos. Além disso, a incidência da doença tem correlação direta com a pobreza. Provavelmente a desnutrição é um fator favorável ao aparecimento da doença, 
mas as péssimas condições de higiene e o fato de várias pessoas se aglomerarem em uma casa e dormirem em um mesmo cômodo aumentam o risco de contágio pelo estreptococo, disparando o processo imunológico em pessoas com sensibilidade para desenvolver $\mathrm{FR}^{5}$. Mediante a isso, soma-se o fato de ser a região Norte a região mais debilitada em questão de educação em saúde .

O Brasil tem sofrido um processo de transição epidemiologica, o declínio do coeficiente de mortalidade geral não é o único aspecto notável no pais nos últimos 50 anos; também a redução da mortalidade infantil, o aumento da expectativa de vida da população, onde a base da piramide etária diminui e há um alargamento do apice da mesma e a modificação do seu perfil epidemiológico foram observados. Este processo, o qual Omran (1971) descreveu como de transição epidemiológica, caracteriza-se pela evolução progressiva de um perfil de alta mortalidade por doenças infecciosas para um outro onde predominam os óbitos por doenças cardiovasculares, neoplasias, causas externas e outras doenças consideradas crónico-degenerativas ${ }^{6}$.

Em 1985, embora as doenças do aparelho circulatório tenham se tornado a primeira causa de morte em todas as regiões brasileiras, quando antes em 1930 eram as doenças infectoparasitarias que ficaram em 10 lugar. Elas correspondiam a $37 \%$ no Sul, Centro-Oeste e Sudeste, a $28 \%$ no Nordeste, e a $22 \%$ na região Norte, enquanto as doenças infecciosas e parasitárias correspondiam a $16 \%$ na região Norte, a $14 \%$ no Nordeste, a $8 \%$ no Centro-Oeste, a apenas $5 \%$ na região Sul, e a $4 \%$ no Sudeste ${ }^{6}$. Assim explica-se o fato da $F R$, uma doença que origina uma valvulopatia que tem distribuição desigual entre as regiões brasileiras, ser mais comum em regiões frias, já que a partir da década de 80 , as doenças circulatórias se tornaram a maior causa de morte no Brasil, concomitante a isso a população de pessoas que se declaram pardas no país é alarmante na região Sul, favorecendo a prevalência de FR E CRC nessa raça humana.

$O$ sexo feminino foi mais acometido em relação o masculino, no entanto não foi encontrado evidências cientificas em que apresente uma explicação para esse predomínio em relação ao masculino, contudo observa-se também que a coréia de Sydenham. Ocorre predominantemente em crianças e adolescentes do sexo feminino. ${ }^{7}$

\section{CONCLUSÃO}

O presente estudo revela que no período de 2012 a 2018 foi observado um total de 268 internações no estado do Tocantins por doença reumática crônica do coração, com maior no ano de 2012, representando $20,8 \%$ do total dos anos analisados. A idade de maior predomínio foi de 40-49 anos, correspondendo a $18,2 \%$ do total analisado, dados que corroboram com a história natural da doença reumática e o período esperado para o aparecimento da cardiomiopatia reumática ${ }^{4}$. De todas as raças apuradas, a parda foi dominante, com 70,5\%, devido as características epidemiológicas do estado, a miscigenação que ocorre no Brasil e em especial no Tocantins. O estudo foi limitado em analisar os dados de número de óbitos, por ter sido analisados apenas 7 anos e não ter sido separar adequadamente os grupos por tempo de evolução do acometimento cardíaco, tendo como $n=12$, que equivale a cerca de $4,5 \%$ de óbitos.

Em decorrência do comprometimento cardíaco entre outras consequências graves, a FR tem que ser uma doença mais difundida no meio médico para o seu reconhecimento precoce e estabelecimento de conduta adequada procurando assim evitar seus agravos. No caso da região norte, esses agravos acarretam ainda mais a morbimortalidade desses pacientes, tanto pela dificuldade diagnóstico em faixas etárias decisivas, como na estrutura complexa para o tratamento da complicação mais associada a FR que são as lesões em válvulas cardíacas $^{2}$.

Apesar das limitações, esta pesquisa traçou o perfil de uma amostra de pacientes ainda pouco estudada, mas necessita de atenção, e trouxe informações relevantes, podendo servir de incentivo a novos estudos mais abrangentes, qualificação e estrutura hospitalar adequada a relevância dessa cardiopatia adquirida no estado.

\section{REFERÊNCIAS}

1- Diretrizes brasileiras para o diagnóstico, tratamento e prevenção da febre reumática. Arq. Bras. Cardiol., São Paulo, v. 93, n. 3, supl. 4, p. 3-18, Sept. 2009. Available from

<http://www.scielo.br/scielo.php?script=sci_arttext\&pid=S 0066-782X2009002100001\&lng=en\&nrm=iso>. access on 26 Feb. 2019. http://dx.doi.org/10.1590/S0066782X2009002100001.

2- Nunes, MC. A cardiopatia reumática - importante causa de cirurgia cardíaca para troca valvar. 2017.

3- Chaves A. A cardiopatia reumática. Arq. Bras. Cardiol. Rio Grande do Sul. P. p. 95-112. 2009. Available from < http://www.cardiol.br/tunel/ago/new/007.pdf>. Access on 26 Feb. 2019

4- SILVA, Carlos Henrique Martins da. Febre reumática: Um estudo multicêntrico no estado de São Paulo. Rev. Hosp. Clin., São Paulo , v. 54, n. 3, p. 85-90, June 1999 Available from

$<$ http://www.scielo.br/scielo.php?script=sci_arttext\&pid=S 0041-87811999000300004\&lng=en\&nrm=iso $>$ access on 18 May 2019. http://dx.doi.org/10.1590/S004187811999000300004.

5- ALVES M. Z. M. et al. Prevalência da Febre Reumática em Crianças de uma Escola da Rede Pública de Belo Horizonte. Arq Bras Cardiol. Minas Gerais, v. 65, no 4, 1995

6- PRATA, P. R. A Transição Epidemiológica no Brasil. Cad. Saúde Públ., Rio de Janeiro, v. 8, n.2, pg 168-175, abr/jun, 1992.

7- PEIXOTO, A. et al. Febre reumática: revisão sistemática. Revista Brasileira de Clinica Medica . São Paulo, 2011 maijun;9(3):234-8.) 